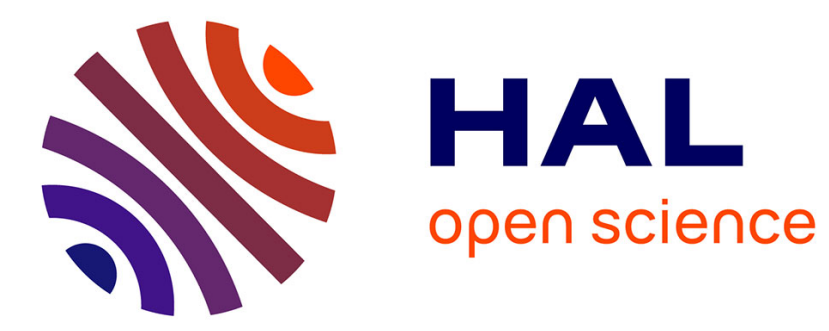

\title{
Langue maternelle et langue seconde : approche par l'observation gestuelle
}

Philippe Turchet

\section{To cite this version:}

Philippe Turchet. Langue maternelle et langue seconde : approche par l'observation gestuelle. Langages, 2013, 192 (4), 10.3917/lang.192.0029 . hal-01756336

\section{HAL Id: hal-01756336 \\ https://hal.science/hal-01756336}

Submitted on 1 Apr 2018

HAL is a multi-disciplinary open access archive for the deposit and dissemination of scientific research documents, whether they are published or not. The documents may come from teaching and research institutions in France or abroad, or from public or private research centers.
L'archive ouverte pluridisciplinaire HAL, est destinée au dépôt et à la diffusion de documents scientifiques de niveau recherche, publiés ou non, émanant des établissements d'enseignement et de recherche français ou étrangers, des laboratoires publics ou privés. 


\section{Philippe Turchet}

Chercheur indépendant en synergologie (Université Paris Ouest Nanterre La Défense \& Université du Québec à Montréal - UQÀM)

\section{Langue maternelle et langue seconde : approche par l'observation gestuelle}

\section{INTRODUCTION}

En situation d'interaction, le langage parlé est généralement accompagné d'un langage gestuel. Or, parler dans sa langue maternelle (L1) ou langue natale et s'exprimer dans une langue seconde (L2) ${ }^{1}$ sont deux attitudes cognitives relevant d'états et de réflexes mentaux et culturels différents (Hewes 1973 ; Steklis \& Harnad 1976 ; Place 2000 ; Skoyles 2000 ; Knecht et al. 2000 ; JacquetAndrieu 2012a). Dans ce contexte, il convient alors de se demander si le fait de s'exprimer en L1 plutôt qu'en L2 induit des variations dans la gestuelle.

Plusieurs typologies, publiées au cours de ces trente dernières années (Ekman \& Friesen 1969 ; McNeill 1992 ; Kendon \& Versante 2003 ; Birdwhistell 1963 ; Cosnier 2006), permettent de dégager des règles de description gestuelle et d'observer la nature culturelle de certains gestes (Morris et al. 1979 ; Caradec 2005 ; Tellier 2006). Toutefois, le choix de la main dominante effectuant les gestes n'a pas encore été classifié de façon systématique dans ces typologies, les auteurs estimant, comme d'autres analyses l'ont montré d'ailleurs (Kimura 1973, 1993 ; Kimura 1981), que la main droite dominante, chez un droitier, est celle avec laquelle les gestes sont généralement effectués, d'autant plus que le rapport neuropsychologique entre le mot et le geste est aujourd'hui mieux connu. En effet, l'aire de Broca $(\mathrm{AB} 44 \& 45)^{2}$, située au pied de la

1. Dans la suite de l'article, nous désignons la langue maternelle (langue natale) et la langue seconde par L1 et L2, respectivement.

2. Les aires corticales des fonctions cognitives sont généralement désignées à partir de la cytoarchitectonie de Brodmann. Il s'agit de la délimitation des types de cellules nerveuses effectuée par Brodmann (1909) ; elles ont été numérotées dans l'ordre de leur étude. 
$3^{\mathrm{e}}$ circonvolution frontale gauche, est déterminante lors de la " production du langage articulé » (Broca 1865) et, dans la mesure où la zone motrice responsable de la gestuelle est contiguë à l'aire de Broca (Cooper 2006 ; Lieberman 2003) et qu'en interaction le geste est pratiquement indispensable à la communication (Goldin-Meadow 1999), il semble bien que le geste joue un rôle de stimulation et de renforcement du langage verbal (Rauscher, Krauss \& Chen 1996 ; Rimé \& Shiaratura 1991). Autrement dit, la proximité de l'aire de Broca et de l'aire gestuelle connexe de l'hémisphère gauche $(\mathrm{HG})$ régissant la partie droite du corps conduirait la main droite à être plus active en situation d'interaction (Steklis \& Harnad 1976 ; Place 2000 ; Skoyles 2000).

En d'autres termes, l'observation systématique de la main dominante devrait permettre d'observer si le choix de la langue parlée, selon qu'il s'agit de L1 ou L2, affecte le choix des mains actives. Si le choix non conscient des mains utilisées était différent, il serait pertinent alors de se poser la question du sens sous-jacent et, notamment, si l'implication émotionnelle au moment de l'interaction doit être prise en compte dans l'explication.

\section{IMPACT DU PASSAGE D'UNE LANGUE MATERNELLE À UNE LANGUE SECONDE SUR LA PRODUCTION GESTUELLE À TRAVERS UN CHOIX D'OBSERVATION ÉCOLOGIQUE}

Plusieurs de nos observations empiriques nous ont conduit à écarter la méthode expérimentale. En effet, et sous réserve d'observations ultérieures, les conditions expérimentales amènent les participants à adopter des comportements contrôlés, quelle que soit l'expérience effectuée. Or, les sphères motrices responsables du contrôle (Wittling 1995), de même que les neurotransmetteurs actifs dans les opérations de mise en œuvre de la vigilance (Tucker \& Williamson 1984), sont majoritairement présents dans l'hémisphère gauche, ce qui, à notre sens, conduit à observer des participants communiquant davantage en conditions expérimentales avec la main droite, que dans des conditions naturelles. C'est pourquoi le choix d'observations en conditions écologiques (aussi naturelles que possible) a été privilégié ici (Cosnier, 2006 : 107), elles peuvent être réellement descriptives.

\subsection{Protocole}

Le choix d'observation vidéo se fonde sur le concept de Comparable Corpus ou cor-

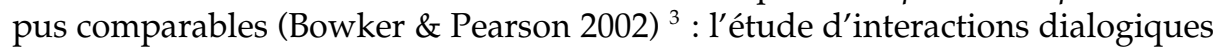
gestuelles de deux sujets parlants du monde de la politique. Le premier (Stephen

3. Comparable Corpus (ou corpus comparable), selon Bowker \& Pearson (2002), c'est une catégorie de corpus comparant des textes oraux ou écrits de deux langues différentes, sans traduction (ici, l'anglais et le français), appartenant à un domaine sémantique très circonscrit (le discours politique) et ayant la même fonction de communication. 
Harper - SH) est Premier ministre au Canada et le second (Gilles Duceppe - GP) était leader de l'opposition francophone en 2011. Ils pratiquent tous deux les deux langues officielles du Canada (l'anglais et le français) ; ils dialoguent avec des journalistes qu'ils connaissent bien.

La langue maternelle du premier (SH), l'anglais, est la langue seconde du deuxième (GP), francophone et vise versa : autrement dit, la L1 de l'un est la L2 de l'autre, les deux corpus centrés sur l'étude du geste sont donc distincts mais comparables.

Pour garantir la pertinence de la comparaison, l'exactitude et la validité des données d'observation collectées, d'autres critères ont été retenus : (i) les deux locuteurs sont des hommes, droitiers, de la même génération ; (ii) ils appartiennent à la même catégorie socioprofessionnelle (CSP) ; (iii) leur niveau dans leur L2 respective est comparable, compte tenu du cadre professionnel dans lequel ils évoluent ; (iv) le fait d'être filmés quotidiennement fait partie de la réalité professionnelle de ces deux hommes publics, ce qui garantit au mieux les conditions dites naturelles, écologiques et non ad hoc des corpus comparés ; (v) la popularité des deux sujets garantissait l'existence de banques de données suffisamment riches pour l'observation.

\subsection{Données brutes du choix des mains en langue maternelle et en langue seconde}

Pour le choix des vidéogrammes, toutes les vidéos électorales et les débats ont été écartés car il faut savoir que la mise sous contrôle de la gestuelle est toujours à craindre dans ces contextes de communication très codifiés. Les vidéos sans interaction ont également été écartées, parce qu'elles ne semblaient pas être disponibles, pour les deux orateurs, pour les deux langues observées. Les publicités télévisuelles partisanes, susceptibles de manquer d'impartialité et de spontanéité, ont également été retirées. Les vidéogrammes finalement sélectionnés (cf. Annexes) correspondent aux situations dans lesquelles les deux sujets parlants étudiés étaient en dialogue avec des animateurs qu'ils connaissent bien ; le climat détendu, propre aux conditions écologiques ou naturelles (Cosnier 2006), était satisfaisant dans les deux contextes linguistiques (anglais et français). Enfin, sur le plan technique, les vidéogrammes ont été intégralement analysés, afin de respecter les conditions d'impartialité et éviter tout biais d'induction (Popper 1934). En outre, il fallait que les durées d'images analysées soient semblables ou approchantes d'un profil d'étude à l'autre.

Les gestes qui sont l'objet principal de cette étude sont strictement délimités : il s'agit du segment gestuel effectué, à partir du moment où la ou les mains prennent la direction et entrent dans l'espace de communication pour l'occuper, jusqu'au moment précis où la/les main(s) retrouve(nt) le corps ou le visage pour s'y poser. Il convient de noter que cette délimitation est proposée en l'absence de convention partagée définissant le geste et permettant de se livrer, à travers les études, à des données d'observation uniformes. 


\subsubsection{Données chiffrées brutes : profil 1 (SH), anglophone (L1) et langue seconde (L2)}

Deux vidéogrammes du profil anglophone (SH) réalisés en L1 (21'57") et

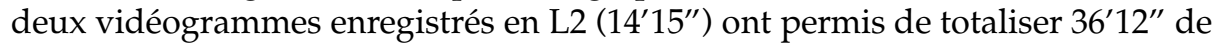
langage gestuel (cf. Annexe 1).

En L1 (l'anglais ici), SH réalise des gestes de la main droite durant 2'32", de la main gauche, durant 47" et des deux mains durant 2'49'. En L2 (le français), il réalise des gestes de la main droite durant $32^{\prime \prime}$, de la main gauche durant 1'21" et des gestes des deux mains durant 2'49'. L'utilisation des deux mains est privilégiée dans les deux langues mais, au moment du passage de L1 à L2, un phénomène marquant apparaît : la forte utilisation des deux mains et la presque disparition de l'utilisation de la main droite.

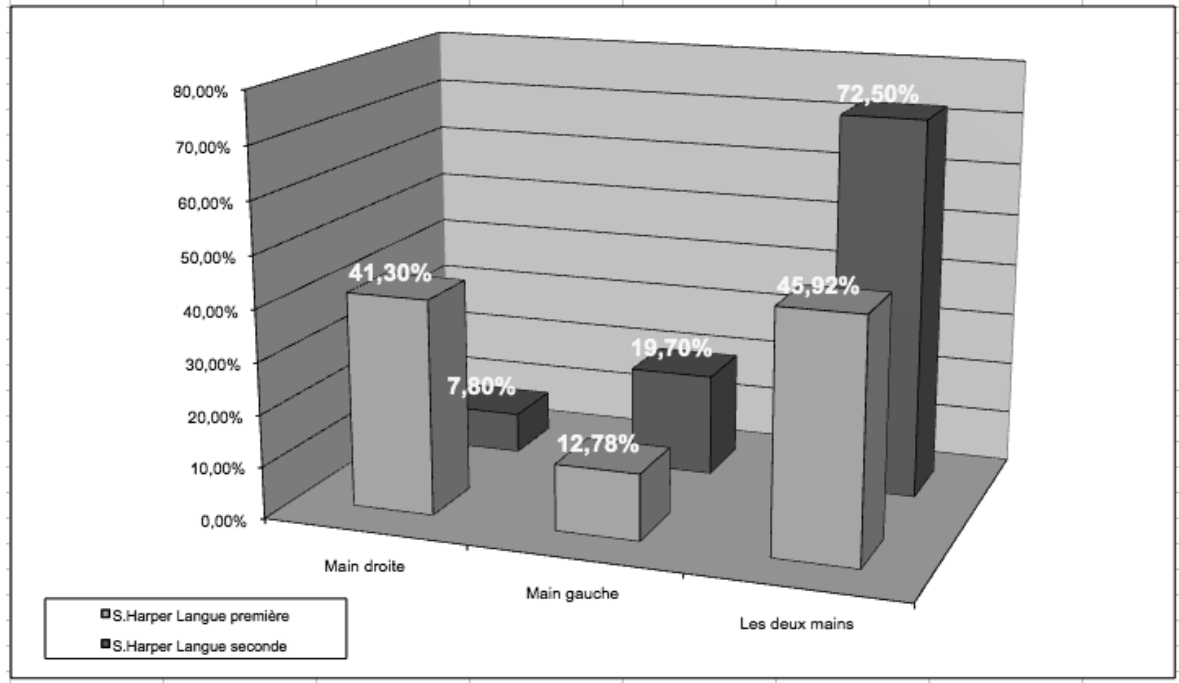

Figure 1 : Données chiffrées de S. Harper

\subsubsection{Données chiffrées brutes : profil 2 (GD), francophone (L1) et langue seconde (L2)}

Deux vidéogrammes réalisés en L1 (18'31") et un vidéogramme en L2 (17'51") ont permis de totaliser 36'22" de langage gestuel (cf. Annexe 2).

En L1 (le français ici), GD réalise des gestes de la main droite durant 1'32', de la main gauche durant $3^{\prime} 23^{\prime \prime}$ et des gestes des deux mains durant 5'24". En L2, il réalise des gestes de la main droite durant $48^{\prime \prime}$, de la main gauche durant 5'31" et des gestes des deux mains durant 7'27'. Là aussi, et dans les deux cas, l'utilisation des deux mains est globalement privilégiée et, plus spécifiquement, 
au passage de L1 à L2. Le phénomène marquant est la presque disparition de l'utilisation de la main droite au profit des deux mains.

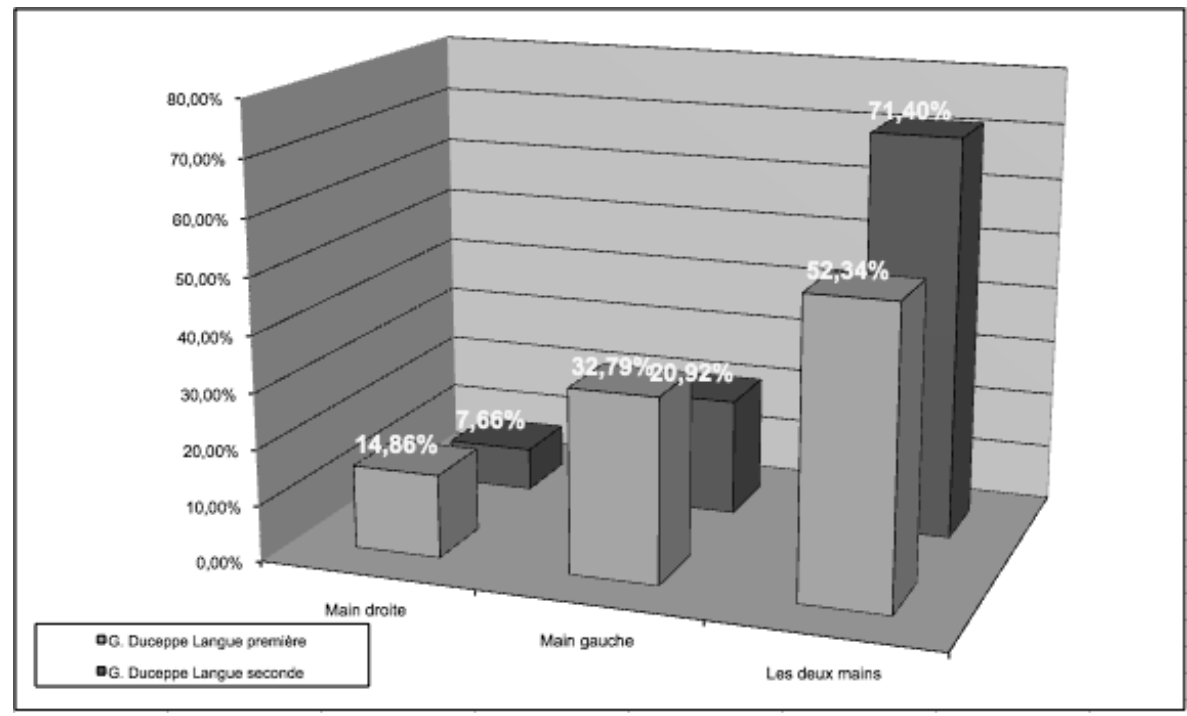

Figure 2 : Données chiffrées de G. Duceppe

\subsection{Observations comparées des deux profils}

La gestuelle des deux sujets parlants semble infirmer les conclusions issues d'études antérieures qui tendent à affirmer que la communication est plus spécifiquement accompagnée de gestes de la main droite en L1 (Bates et al. 1986 ; Bellugi 1991 ; Blake, O’Rourke \& Borzellino 1994). En effet, si l'on compare l'activité des deux mains, SG (anglophone) communique davantage avec la main droite en L1 (ratio : 3.23) ; à l'inverse, GD (francophone) communique davantage avec la main gauche en L1 (ratio : 2.20), alors qu'il est droitier. Ces observations sont conformes à nos observations antérieures ${ }^{4}$.

Dans le passage de L1 à L2, maintenant, un même transfert gestuel a lieu chez les deux sujets. Il se traduit par un langage gestuel effectué plus essentiellement avec les deux mains, lorsque les locuteurs passent de leur L1 à leur L2, respectivement. En L2, le locuteur anglophone, alors qu'il est droitier, utilise sa main droite durant moins de $10 \%$ du temps. S'il y a un rapport direct entre l'aire de Broca (AB 44 \& 45), le langage (Decety et al. 1997 ; Grèzes, Costes \& Decety 1999 ; Zatorre et al. (1992) ; Gallagher \& Frith 2004) et la main droite, les 
profils devraient avoir davantage besoin de stimuler les zones sémantiques en L2 et utiliser le ressort de la main droite. Or, les observations présentes ne vont pas dans ce sens. Si l'élargissement de cette étude devait confirmer ces résultats, il faudrait poser autrement le rapport entre langage verbal et langage gestuel (Turchet 2009).

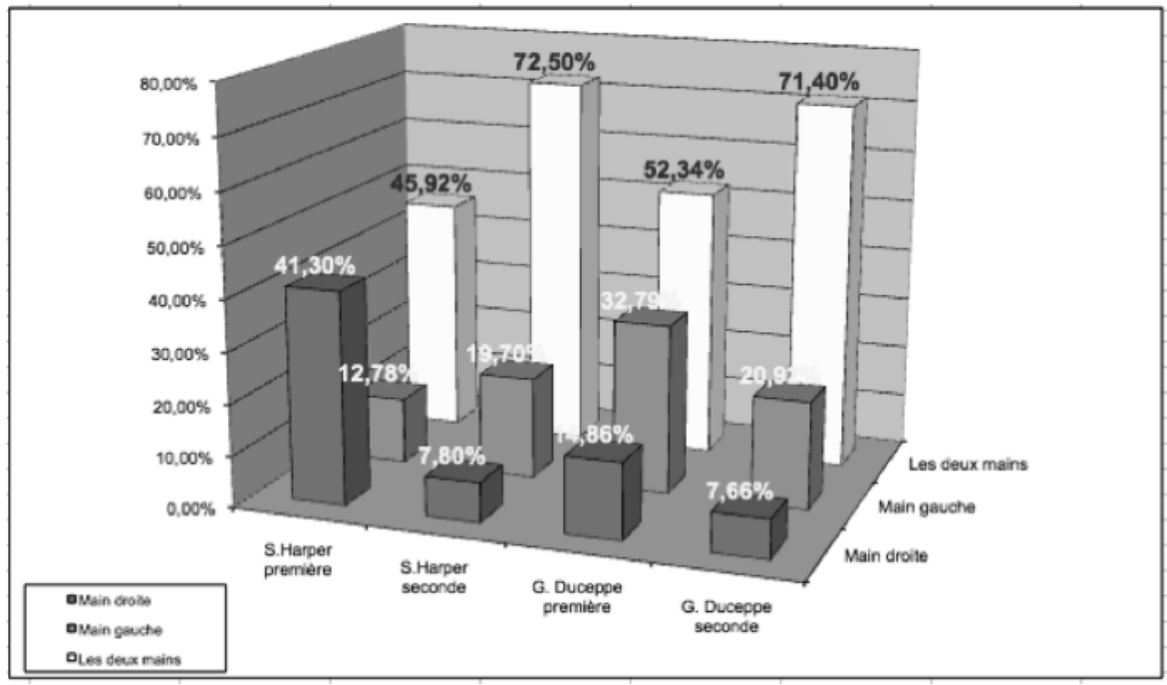

Figure 3 : Données comparées

\subsection{Discussion préliminaire et premières hypothèses}

Il est possible qu'en L2, le déplacement gestuel vers une communication intéressant les deux mains traduise le fait que le corps éprouve la nécessité d'être davantage impliqué lorsque les participants parlent une langue moins bien mâ̂trisée (L2). Ce changement pourrait être dû, en partie, à l'instabilité liée au fait de chercher ses mots, comme si le corps suppléait le déficit sémantique (l'anomie), en occupant davantage le terrain de la communication, avec l'apport de gestes et, ce, afin de mieux occuper l'espace de l'attention mutuelle, partagée par les deux locuteurs (Bates 1976 ; Camaiomi et al. 2004 ; Jacquet-Andrieu 2012a), proposer à l'autre de coopérer (Liszkowski 2005).

Dans la littérature, on trouve une classification qui permet de mieux rendre compte des observations effectuées. Elle oppose deux types de gestes : les gestes impératifs (Bates 1976) et les gestes déclaratifs (Camaiomi et al. 2004 ; Liszkowski 2005). Les premiers ne sont pas spécifiques à l'humain, certains primates non humains les partagent (Tomasello 1996 ; Meguerditchian \& Vauclair 2009 ; Gomez 2007) ; ils sont qualifiés d'universels et, globalement, ils sont réalisés de la main droite, aussi bien chez le primate non humain que chez 
l'homme ; ils pourraient être à l'origine du langage verbal (Corina et al. 2003 ; San José-Robertson et al. 2004). Ces gestes, réalisés à partir de connexions cérébrales connexes à l'aire de Broca, dans l'hémisphère gauche (Emmorey, Mehta \& Grabowski 2007 ; Kimura 1973 ; Dalby et al. 1980 ; Kimura \& Humphrys 1981 ; Lavergne \& Kimura 1987 ; Meguerditchian \& Vauclair 2009), pourraient expliquer que la gestuelle de la main droite soit plus présente.

En L2, il est possible que, pour maintenir l'attention focalisée de l'interlocuteur, face à un déficit possible de la production sémantique (une anomie, par exemple), les gestes déclaratifs fassent leur apparition dans l'espace de communication (Kendon \& Versante 2003 ; Wilkins 2003). Ces gestes, pour lesquels la main gauche apparaîtrait à côté de la main droite, seraient eux réalisés sans intention spécifiquement identifiée, hormis l'intentionnalité, la volonté de communiquer. Ils seraient plus liés à l'activité de l'hémisphère droit ; ce dernier, souvent identifié comme étant l'hémisphère de l'émotionnel (Bourassa 1997 ; Buser 1998 ; Rinn 1995 ; Tucker \& Williamson 1984), mais aussi de l'intuition (Cadet \& Jacquet-Andrieu 2012), serait plus actif à cette occasion (Meguerditchian 2009) ${ }^{5}$.

Dans les moments de communication difficiles, le corps pourrait ainsi suppléer les «manques ", en intervenant davantage, et il recourrait inconsciemment aux deux mains qui apparaîtraient ensemble, dans l'espace de communication, comme il le fait, par exemple, lorsqu'il traduit son impuissance en haussant les épaules ou en levant les bras au ciel, et ce, dans la plupart des cultures (Morris 1977 ; Caradec 2005). Cependant, il est possible aussi que l'explication soit plus nuancée et que, lors du passage d'une langue parfaitement maîtrisée à une autre qui l'est moins, un changement d'état émotionnel plus global (Damasio 2010) soit lui-même le vecteur principal du changement de la main active. Si c'était le cas, une manière efficace de vérifier cette hypothèse serait de se centrer sur les moments où la production du langage gestuel est atypique, où le choix de la ou des mains actives est inattendu, et d'évaluer alors les émotions éveillées par le thème abordé, en vue de repérer si elles contribuent ou non à produire le changement de main(s) active(s) dans l'interaction. La preuve serait faite alors que, dans leur surgissement inconscient, les émotions sont plus impliquées que l'on a souvent tendance à l'estimer, dans la production gestuelle (Jacquet-Andrieu 2012b) et, surtout, l'observation du langage gestuel pourrait être un indicateur pertinent pour en rendre compte (Turchet 2009, 2013). Nous proposons, à la suite, une autre observation allant dans ce sens.

5. Rappelons ici que le circuit des émotions se déploie en un réseau complexe qui, avec le tronc cérébral, implique aussi des structures cortico-sous-corticales (Damasio 2010). 


\section{IMPACT DE L'ÉMOTION SUR LA PRODUCTION GESTUELLE : OBSERVATIONS COMPLÉMENTAIRES}

Les changements d'état mental au passage de L1 à L2 sont observables à la modification du langage gestuel. Les émotions impliquées dans la modification des circuits neuronaux pourraient être, en partie du moins, responsables de ce phénomène. Rien en tout cas ne permet de le nier, a priori. Mais, si la dynamique du geste est liée à l'émotion, un thème de discussion à forte teneur émotionnelle devrait, par lui-même, être susceptible de modifier la production gestuelle. L'émotion est-elle lisible ou non, à travers la production gestuelle?

Il s'avère que, sur un vidéogramme, le locuteur anglophone dialogue avec le même journaliste à la fois en français (L2) et en anglais (L1), alternant les réponses dans l'un ou l'autre idiome. Il nous a semblé pertinent d'analyser ces images car elles permettent d'affiner les réflexions qu'apporte la première série d'observations, surtout que les conditions d'observation restent stables, durant ces passages d'une langue à l'autre.

\subsection{D'une langue maternelle à une langue seconde : observations complémentaires}

Dans cette nouvelle interaction entre SH (profil anglophone) et un animateur, ce dernier fait le lien entre des questions vidéogrammes d'internautes (40'32'), réagissant dans les deux langues (cf. Annexe 2). L'animateur a posé douze questions, mais la dynamique de la réponse à la première a éveillé notre attention car, à cette question (Q1), la réponse a eu lieu d'abord en français (L2 de SH : 1'52'), puis (Q1bis), en anglais (L1 de SH : 1'30'). C'est la seule question à laquelle le locuteur a répondu dans les deux langues : L2 puis L1.

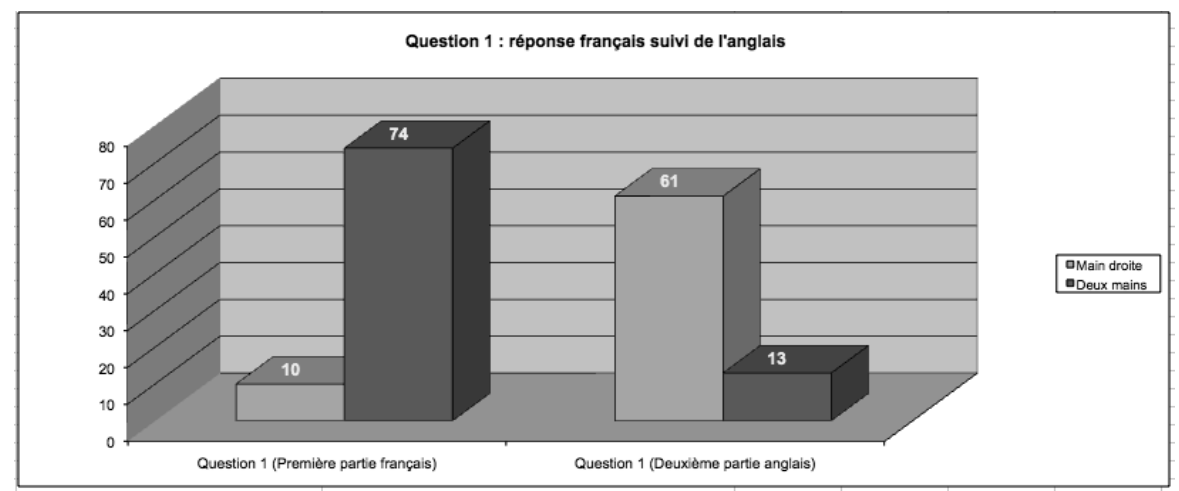

Figure 4 : Questions complémentaires 
Les gestes de la main gauche totalisent moins de 6" $(0.27 \%)$ de la durée du vidéogramme. Les données de la main gauche ont donc été exclues des schémas proposés, pour une meilleure lisibilité des graphiques. Sur les réponses 1 et 1 bis qui sont l'objet du graphique supra, la main gauche est en l'occurrence totalement absente. Le locuteur SH s'exprime d'abord en L2, avant de terminer en L1. Le phénomène observé, mesuré en secondes, montre un changement radical : le locuteur passe de L2 à L1, en modifiant profondément sa gestuelle : des deux mains, en L2, il passe à la main droite pratiquement seule, en L1.

Ce déplacement gestuel est conforme à la logique des observations de la première partie de l'étude. Avant même d'aller aux conclusions, notons que l'observation tendant à dire que davantage de gestes sont visibles en L2 (Gullberg 1998 ; Gullberg \& McCafferty 2008 ; Yoshioka 2005) ne se donne pas à voir sur ce vidéogramme ni dans les précédents d'ailleurs. Ici, la portion de message verbal, dite en L2 (1'49"), est occupée par 1'24" de gestes, soit $77 \%$ de la durée totale, alors que, dans la portion en L1 (1'16"), les gestes couvrent $1^{\prime} 14^{\prime \prime}$, soit $97 \%$ de la durée. La portion gestuelle observée est donc plus importante quand la personne parle en L1, même si la gestuelle ne semble pas forcément plus visible car le locuteur utilise une seule main en L1.

\subsection{Proposition inductive visant à lier distribution du geste et nature de l'émotion}

Pour le reste de l'analyse, les réponses de $\mathrm{SH}$, en anglais (L1) et en français (L2), ont fait l'objet de deux traitements différents. Une méthode inductive a été utilisée pour cibler, au mieux, ce qui pourrait être atypique dans les réponses, et voir si la variable de l'état émotionnel peut être impliquée dans des variations gestuelles inattendues.

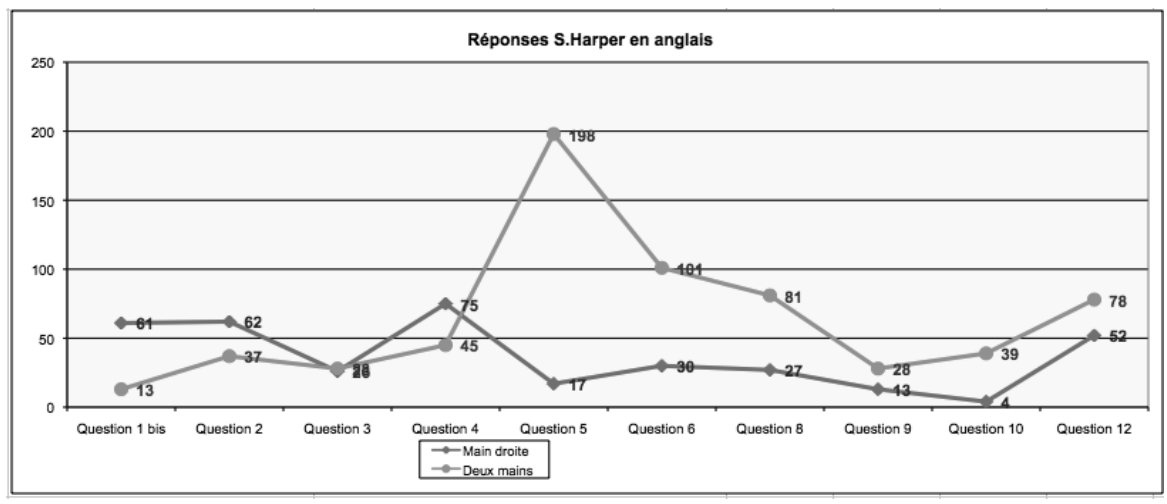

Figure 5 : Réponses en anglais de S. Harper 


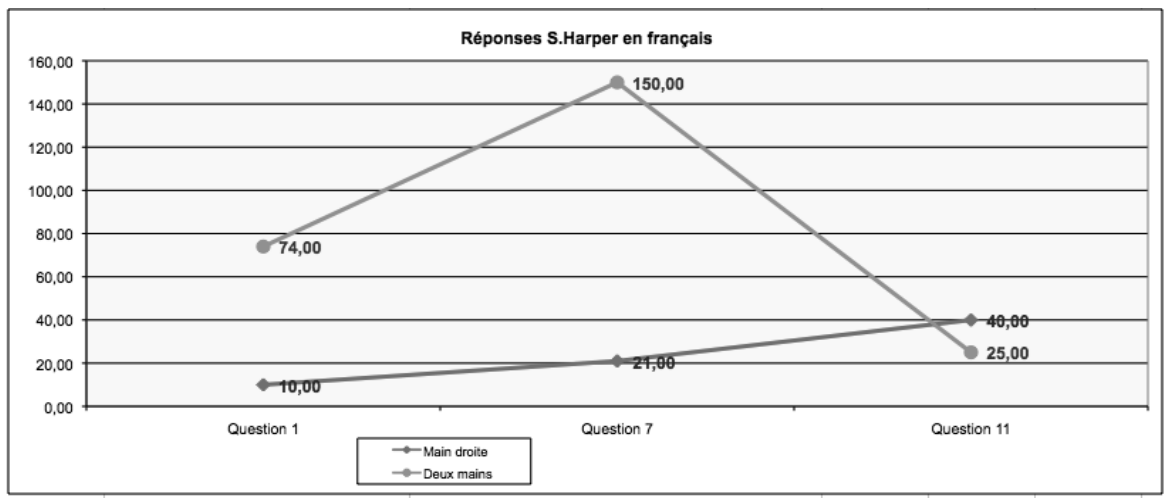

Figure 6 : Réponses en français de S. Harper

Conformément aux observations précédentes, les deux mains sont plus utilisées en L2 (78 \%) qu'en L1 (66 \%). Là encore, tout se passe comme si le corps venait suppléer la parole, dans les situations où la personne manque d'assurance, et comme si la main droite était plus sollicitée en L1 qu'en L2 mais, surtout, la participation gestuelle semble atypique dans deux réponses : la réponse 5 , en $\mathrm{L} 1$, et la réponse 11, en L2. La nature émotionnelle du thème abordé auraitelle contribué à modifier la distribution gestuelle, lors des réponses à ces deux questions?

Pour la réponse à la question 5, L1 du locuteur, la question montre une sur-participation atypique des deux mains : elles interviennent près de 12 fois plus longtemps $(11,64)$ que la main droite alors que la moyenne est proche de la parité $(0.60)$ pour les autres réponses confondues. L'impact émotionnel du thème évoqué expliquerait-il un mal-être ou une instabilité, responsables alors de la surreprésentation des deux mains ? Comme nous l'avons déjà évoqué, les deux mains sont plus présentes dans les situations difficiles. Or, la question 5 est une question sur le réchauffement climatique ; elle a effectivement un statut particulier. La politique énergétique du Canada a beaucoup évolué depuis l'accession au pouvoir de S. Harper (SH, le profil observé). Et lui-même fait l'objet d'une poursuite intentée en Cour fédérale (janvier 2012) demandant que le retrait du Canada du protocole de Kyoto soit déclaré illégal. Si un jour, un lien de cause à effet, plus clair encore, était établi entre des mesures environnementales inadaptées et le réchauffement climatique, il serait personnellement contraint de prendre sa part de responsabilité dans cet état de fait. Des douze questions posées, celle-ci est, de loin, la plus embarrassante, celle dont la réponse est la plus difficile pour le premier ministre. La surreprésentation des deux mains semble permettre d'aller dans le sens de la validation de l'hypothèse selon laquelle le doute ou le mal être se traduisent ainsi, dans cette réponse. 
L'observation des trois réponses en langue française (L2) permet également de voir que le comportement gestuel de ce locuteur est atypique pour la question 11, à laquelle ce dernier répond en L2 et mobilise plus la main droite. La production gestuelle favorable à une main droite dominante se donnant à voir plus volontiers en L1 qu'en L2, il convient de s'interroger sur la nature de la question posée : sa teneur émotionnelle procurerait-elle au locuteur un surcroît d'assurance significatif?

Il s'agit d'une question sur la souveraineté du Québec, sur son indépendance, en fait. Ici le premier ministre, chef du parti conservateur du Canada, est vraiment à l'aise et répond que, constitutionnellement, il pourrait déclencher un référendum, mais qu'il n'en fera rien. Il est d'autant plus à l'aise que son électorat traditionnel est défavorable au dit référendum : il est lui-même contre et une partie de sa popularité provient de cette position. Sa réponse est sans ambiguïté, catégorique, c'est celle d'un chef qui dit «non » et il utilise davantage la main droite.

\section{CONCLUSION}

Dans l'attente d'autres réponses, les dernières observations vidéos, en lien avec les précédentes, autorisent à penser qu'un changement de langage gestuel pourrait être l'expression d'une modification de l'état mental du locuteur, liée à un changement de la teneur émotionnelle de la situation de dialogue. Deux attitudes corporelles distinctes peuvent être mises en exergue : la première consisterait à s'exprimer davantage avec les deux mains en climat d'instabilité, alors qu'en climat d'assurance ou même de force - ce qu'il y aurait lieu d'évaluer plus précisément -, la personne utiliserait davantage la main droite. Par ailleurs, si au passage d'un idiome à un autre, l'impact émotionnel était vérifiable dans les termes énoncés ici, l'observation gestuelle devrait aider le didacticien des langues à différencier un apprenant sûr de lui, communiquant avec une main dominante seule, de celui qui comblerait un déficit de vocabulaire et/ou d'assurance, par une faconde gestuelle surabondante. La maîtrise de la situation se donnant à voir au moment du passage d'une gestuelle des deux mains à une seule, active.

Ces types d'observations, s'ils étaient systématisés, seraient d'un intérêt majeur pour les didacticiens des langues. Ils permettraient de comprendre, grâce à la gestuelle, à partir de quel moment un locuteur, bien que parlant en L2, a acquis une assurance comparable à celle que lui permet la pratique de sa langue maternelle (L1). Il conviendrait alors d'opérationnaliser cette donnée pour que les enseignants puissent la prendre en compte dans l'évaluation des apprentissages et la détermination de leur progression. Par ailleurs, la production gestuelle pourrait être un indicateur pertinent de l'état émotionnel du sujet parlant durant sa gestuelle. Plus généralement, peut-être une meilleure connaissance de l'homme dans sa position de sujet parlant commence-t-elle là. 


\section{Références}

BATES E. (1976), Language and Context. The acquisition of pragmatics, New York: Academic Press.

BATES E. et al. (1986), “Language and hand preference in early development”, Developmental Neuropsychology 2, 1-15.

BELLUGI U. (1991), “The link between hand and brain: Implications from a visual language", in D. S. Martin (ed.), Advances in Cognition, Education and Deafness, Washington (DC): Gallaudet University Press, 11-35.

BIRDWHISTELL R. L. (1963), “The kinesic level in the investigation of the emotions", in P. H. Knapp (ed.), Expression of the Emotions in man, New York: Academic Press, 11-89.

BLAKE J., O'Rourke P. \& Borzellino G. (1994), "Form and function in the development of pointing and reaching gestures", Infant Behavior \& Development 17 (2), 195-203.

BOURASSA M (1997), "Le profil fonctionnel : les apports de la neuropsychologie à l'adaptation scolaire ", Éducation et francophonie XXV (2). [http://www.acelf.ca/c/revue/revuehtml/252/r252-01.html]

BOWKER L. \& PEARSON J. (2002), Working with Specialized Language. A Practical Guide to Using Corpora, London/New York: Routledge.

Broca P. (1865), "Sur le siège de la faculté du langage articulé ", Bulletin de la Société d'Anthropologie de Paris 6, 377-393.

Brodmann K. (1909), Lokalisationslehre der Grosshirnrinde, Leipzig : J. A. Barth.

BUSER P. (1998), Cerveau de soi, cerveau de l'autre, Paris : Odile Jacob.

CADET B. \& JACQUet-ANDRIEU A. (2012), "Émotions, langage et prises de décision : un réseau cognitif ", Holism and Health, 22-29. [http://journal.celenie.ru/index.php/cadet-jacquetandrieu-emotions]

CAMAIONI L. et al. (2004), "The Role of Declarative Pointing in Developing a Theory of Mind", Infancy 5 (3), 291-308.

CARADEC F. (2005), Dictionnaire des gestes : attitudes et mouvements expressifs en usage dans le monde entier, Paris : Fayard.

COOPER D. L. (2006), "Broca's arrow: evolution, prediction and language in the brain”, The Anatomical Record New Anatomy 289 (1), 9-24.

CoRINA D. P. et al. (2003), “Language lateralization in a bimanual language”, Journal of Cognitive Neuroscience 15, 718-730.

CosNier J. (2006), Psychologie des émotions et des sentiments, Paris : Retz.

DALBY J. T. (1980), “Lateralized hand gesture during speech", Journal of Motor Behavior 12, 292-297.

DAmAsio A. R. (2010), L'Autre moi-même. Les nouvelles cartes du cerveau, de la conscience et des émotions, Paris : Odile Jacob.

DECETY J. et al. (1997), "Brain activity during observation of actions: Influence of action content and subject's strategy", Brain 120, 1763-1777.

EKMAN P. \& FRIESEN W. (1969), “The repertoire of non verbal behavior, categories, origins, usage and coding", Semiotica 1, 49-98.

Emmorey K., Mehta S. \& Grabowski T. J. (2007), “The neural correlates of sign versus word production", Neuroimage 36, 202-208.

GaLlagheR H. L. \& FRITH C. D. (2004), "Dissociable neural pathways for the perception and recognition of expressive and instrumental gestures”, Neuropsychologia 42, 1725-1736. 
Goldin-Meadow S. (1999), "The role of gesture in communication and thinking", Trends in Cognitive Sciences 3, 419-429.

GOMEZ J. C. (2007), "Pointing behaviors in apes and human infants: a balanced interpretation", Child Development 78, 729-734.

GRÈzes J., Costes N. \& DECETY J. (1999), "The effects of learning and intention on the neural network involved in the perception of meaningless actions", Brain 122, 1875-1887.

GULLBERG M (1998), Gesture as a communication strategy in second language discourse. A study of learners of French and Swedish, Lund: Lund University Press.

GULLBERG M. \& MCCAFFERTY S. G. (2008), “Introduction to gesture and SLA: Toward an integrated approach", Studies in Second Language Acquisition 30, 133-146.

HEWES G. W. (1973), "Primate communication and the gestural origin of language”, Current Anthropology 14, 5-24.

JACQUET-ANDRIEU A. (2012a), Langage de l'homme. De l'étude pluridisciplinaire à l'action transdisciplinaire, Sarrebruck : Presses Académiques Francophones.

JACQUET-ANDRIEU A. (2012b), "Entre langage \& émotion : de Saussure à Coseriu ", Les langues latines et l'interculturalité.

[http://hal.inria.fr/docs/00/73/27/54/PDF/JACQUET_COLLOQUE_MOSCOU2012.pdf]

Kendon A. \& Versante L. (2003), "Pointing by hand in Neapolitan", in S. Kita (ed.), Pointing: where language, culture, and cognition meet, Mahwah (NJ): Lawrence Erlbaum Associates, 109-137.

KIMURA D. (1973), “Manual activity during speaking: I. Right-handers”, Neuropsychologia 11, 45-50.

KIMURA D. (1993), Neuromotor mechanisms in human communication, Oxford: Oxford University Press.

KIMURA D. \& HUMPHRYS C. A. (1981), "A comparison of left and right-arm movements during speaking”, Neuropsychologia 19, 807-812.

KNECHT S. et al. (2000), "Handedness and hemispheric language dominance in healthy humans", Brain 123, 2512-2518.

LAVERGNE J. \& KIMURA D. (1987), "Hand movement asymmetry during speech. No effect of speaking topic", Neuropsychologia 25, 689-693.

LieBerman P. (2003), "On the Nature and Evolution of the Neural Bases of Human Language", Yearbook of physical anthropology 45, 36-62.

LISZKowskI U. (2005), "Human twelve-month-olds point cooperatively to share interest with and provide information for a communicative partner", Gesture 5, 135-154.

MCNEILL D. (1992), Hand and mind: What gestures reveal about thought, Chicago: University of Chicago Press.

MorRIS D. (1977), Manwatching. A Field Guide to Human Behavior, NewYork: H. N. Abrams.

MorRIS D. et al. (1979), Gestures: their origin and distribution, New York: Stein and Day.

Meguerditchian A. (2009), Latéralité et communication gestuelle chez le babouin et le chimpanzé : à la recherche des précurseurs du langage, Thèse de doctorat de l'Université de Provence.

MEgUERDITCHIAN A. \& VAUCLAIR J. (2009), "Contrast of hand preferences between communicative gestures and non-communicative actions in baboons: implications for the origins of hemispheric specialization for language", Brain and Language 108 (3), 167-174.

PLACE U. T. (2000), "The role of the hand in the Evolution of Language", Psycholoquy 11 (7) Language Gesture (1). [http://www.cogsci.ecs.soton.ac.uk/cgi/psyc/newpsy?11.007] 
POPPER K. (1934), La logique de la découverte scientifique, Paris : Payot.

Rauscher F. H., Krauss R. M. \& Chen Y. (1996), “Gesture, speech, and lexical access: The role of lexical movements in speech production”, Psychological Science 7, 226-231.

Rimé B. \& Shiaratura L. (1991), “Gesture and speech”, in R. S. Feldman \& B. Rime (eds), Fundamentals of non- verbal behaviour, Cambridge: Cambridge University Press, 239-281.

RINN W. (1995), "The neurospsychology of facial expressions: a review of neurological and the psychological mechanisms of producing facial expressions", Psychological Bulletin 95, 52-77.

SAN JOSE-ROBERTSON L. (2004), “Neural systems for sign language production: mechanisms supporting lexical selection, phonological encoding, and articulation", Human Brain Mapping 23 (3), 156-167.

SKoYLES J. R. (2000), “Gesture, language origins, and right handedness”, Psycoloquy 11 (24) Language Gesture (2). [http://www.cogsci.ecs.soton.ac.uk/cgi/psyc/newpsy?11.024]

SteKLIS H. D. \& HARNAD S. (1976), "From hand to mouth: Some critical stages in the evolution of Language", Annals of the New York Academy of Sciences 280, 445-455.

TELLIER M. (2006), L'impact du geste pédagogique sur l'enseignement-apprentissage des langues étrangères. Étude sur des enfants de 5 ans, Thèse de doctorat de l'Universitéi Paris 7 - Denis Diderot.

Tomasello M. (1996), “Do apes ape?”, in B. Galef \& C. Heyes (eds), Social learning in animals: The roots of culture, San Diego: Academic Press, 319-346.

TUCKER D. M. \& WiLliamSON P. A. (1984), “Asymmetric neural control systems in human selfregulation", Psychological Review 91 (2), 185-215.

TURCHET P. (2009), Le langage universel du corps. Comprendre l'être humain à travers la gestuelle, Montréal (Québec) : Les Éditions de l'Homme.

TURCHET P. (2013), "Préalables épistémologiques à une théorisation du langage corporel ", in A. Jacquet-Andrieu (éd.), Sous-jacentes au langage, les émotions, Limoges : Lambert Lucas (à par.).

WILKINS D. (2003), "Why pointing with the index finger is not a universal (in sociocultural and semiotic terms)", in S. Kita (ed.), Pointing: Where language, culture, and cognition meet, Mahwah (NJ): Lawrence Erlbaum Associates, 171-215.

WITTLING W. (1995), "Brain asymmetry in the control of autonomic-physiologic activity", in R. J. Davidson \& K. Hugdahl (eds), Brain Asymmetry, Cambridge (MA): The MIT Press, 305-357.

YosHIOKA K. (2005), Linguistic and gestural inroduction and tracking referrents in L1 and L2 discourse, Groningen: Groningen Dissertations in Linguistics 55.

ZATORRE R. J. et al. (1992), "Lateralization of phonetic and pitch discrimination in speech processing”, Science 256, 846-849.

\section{Annexes}

\section{Annexe 1 - S. Harper}

- Vidéogrammes anglophones

- CBC. The National. Entretien avec P. Mansbridge. CBC, 08.09.2011

- CBC. The National. Entretien avec P. Mansbridge. 01.2012

- Vidéogrammes francophones

- Radio Canada. Téléjournal. Entretien avec E. Latraverse. 16.01.2012

- Radio Canada. Télé journal. Entretien avec D. Lessard. 28.04.2011 
- Vidéogramme supplémentaire

- Youtube. Entretien avec P. Pichette. 2011

Annexe 2 - G. Duceppe

- Vidéogrammes francophones

- Radio Canada. Tout le monde en parle. Entretien avec G. A. Lepage. 11.04.2011

- Canal V. Entretien avec Mario Dumont. 27.04.2011

- Vidéogramme anglophone

- CBC. The Hour. Entretien avec G. Stroumboulopoulos. 8.04.2010 